\title{
Neighborhood environment and muscle mass and function among rural older adults: a 3-year longitudinal study
}

Kenta Okuyama ${ }^{1,2^{*}} \mathbb{D}$, Takafumi Abe ${ }^{2}$, Shozo Yano ${ }^{2,3}$, Kristina Sundquist $^{1,2,4}$ and Toru Nabika ${ }^{2,5}$

\begin{abstract}
Background: Sarcopenia, resulting from loss of muscle mass and function, is highly prevalent in the ageing societies and is associated with risk of falls, frailty, loss of independence, and mortality. It is important to identify environmental risk factors, so that evidence-based interventions to prevent sarcopenia can be implemented at the population level. This study aimed to examine the potential effect of several objectively measured neighborhood environmental factors on longitudinal change of muscle mass and function among older adults living in rural Japanese towns where the population is ageing.

Methods: This study was based on data from the Shimane CoHRE Study conducted by the Center for Communitybased Healthcare Research and Education (COHRE) at Shimane University in 3 rural towns in the Shimane Prefecture, Japan. Subjects older than 60 years, who participated in an annual health examination in 2016 and any follow-up years until 2019, i.e., 4 possible time points in total, were included $(n=2526)$. The skeletal muscle mass index (SMI) and grip strength were assessed objectively for each year as a measure of muscle mass and function, respectively. Neighborhood environmental factors, i.e., hilliness, bus stop density, intersection density, residential density, and distance to a community center were measured by geographic information systems (GIS). Linear mixed models were applied to examine the potential effect of each neighborhood environmental factor on the change of SMI and grip strength over time.
\end{abstract}

Results: Males living far from community centers had a less pronounced decline in SMI compared to those living close to community centers. Females living in areas with higher residential density had a less pronounced decline in grip strength compared to those living in areas with lower residential density.

Conclusions: Neighborhood environmental factors had limited effects on change of SMI and grip strength among rural older adults within the 3 years follow up. Further long-term follow up studies are necessary by also taking into account other modifiable neighborhood environmental factors.

Keywords: Neighborhood environment, Sarcopenia, SMI, Grip strength, Rural

*Correspondence: kenta.okuyama@med.lu.se

${ }^{1}$ Center for Primary Health Care Research, Lund University, Jan Waldenströms gata 35, 20502 Malmö, Sweden

Full list of author information is available at the end of the article

\section{Introduction}

Sarcopenia is defined as a progressive and generalized skeletal muscle disorder resulting from loss of muscle mass and muscle function and is associated with risk of falls, frailty, loss of independence, and mortality among older adults [1-4]. Several factors, such as physical activity, diet, and smoking have been identified as potential risk factors for sarcopenia [5-8]. While these individual

c) The Author(s) 2020. This article is licensed under a Creative Commons Attribution 4.0 International License, which permits use, sharing, adaptation, distribution and reproduction in any medium or format, as long as you give appropriate credit to the original author(s) and the source, provide a link to the Creative Commons licence, and indicate if changes were made. The images or other third party material in this article are included in the article's Creative Commons licence, unless indicated otherwise in a credit line to the material. If material is not included in the article's Creative Commons licence and your intended use is not permitted by statutory regulation or exceeds the permitted use, you will need to obtain permission directly from the copyright holder. To view a copy of this licence, visit http://creativeco mmons.org/licenses/by/4.0/. The Creative Commons Public Domain Dedication waiver (http://creativecommons.org/publicdomain/ zero/1.0/) applies to the data made available in this article, unless otherwise stated in a credit line to the data. 
modifiable factors are important in interventions at the individual level, neighborhood-level factors may also be key in the prevention of sarcopenia at the population level because of their potential to determine people's behaviors and subsequent health outcomes. Neighborhood environments may be of importance particularly for older adults, who are likely to spend more time in their neighborhoods of residence than younger persons [9]. Several studies have examined the association between neighborhood environmental factors, e.g., safety, physical disorder (trash or littering), and social cohesion and older adults' physical functions such as activities of daily living [10-14]. One study from the US found that living in neighborhoods with high physical disorder, low social cohesion and low safety was associated with higher incidence of limitations in activities of daily living, such as getting across the room, bathing, eating and shopping for groceries [14]. Other studies from the UK and Australia have found that living in neighborhoods with low socioeconomic status is associated with poorer physical function measured by self-reported functional limitations in different daily activities [10-12, 15]. One study from the US assessed physical function objectively and found that self-reported neighborhood disorder was associated with lower physical function [13]. However, no previous studies have assessed both neighborhood environments and physical functions based on objective measures, such as functions related to sarcopenia. Sarcopenia has been defined as a clinical disorder in WHO's International Classification of Diseases (ICD) version 10 (ICD-10-CM (M62.84)) in 2016 and is based on objectively measured muscle mass and function [16]. There is firm evidence that these objective measures are associated with falls, frailty, loss of independence, and mortality [17-22]. Given this, studies should also strive to identify neighborhood environmental factors that may affect objectively measured muscle mass and function, in order to find avenues for prevention and environmental interventions to avoid sarcopenia at the population level. Sarcopenia is currently estimated to amount to a prevalence of $10-40 \%$ and is therefore associated with high healthcare costs $[23,24]$. Current findings for neighborhood environmental factors associated with physical function are also limited to urban western settings with a crosssectional design [25]. Furthermore, while its association might differ by gender, few studies have analyzed males and females separately and with a sufficient sample size.

Considering this background, our study aimed to investigate whether objectively measured neighborhood environmental factors may affect objectively measured muscle mass and function among older adults by using a longitudinal study design within rural towns of Japan where the population is ageing.

\section{Methods}

\section{Study design}

This 3-year longitudinal study, using data from the Shimane CoHRE Study, was conducted by Shimane University in collaboration with 3 municipalities in Shimane Prefecture. The Shimane CoHRE Study aims to prevent lifestyle-related diseases by collecting data on medical information, lifestyle, physical function, and neighborhood environment during annual municipal health examinations. The present study used data between 2016, when the study began and 2019 to measure muscle mass and function. Informed consent was obtained from all subjects before enrolment. This study was conducted with the approval of the Ethics Committee of Shimane University School of Medicine (\#20180420-2).

\section{Study subjects}

Rural mountainous regions of Japan, which feature a unique environment, with a proportion of an older adult population above approximately $35 \%$ were subject in this study. Subjects were older adults over the age of 60 years residing in the towns of Ohnan, Okinoshima, and Unnan in the Shimane Prefecture as shown in the maps in Figs. 1, 2, 3, and 4. Eligibility for this study was participation in the 2016 baseline survey, which measured muscle mass and function and participation in any of the followup surveys during the 3 years between 2017 and 2019 . The final analysis included 2526 subjects (1013 males and 1513 females). Exclusion was performed based on the following criteria: 43 subjects with missing skeletal muscle mass index (SMI) values at baseline, 13 subjects with missing grip strength values at baseline, and 30 subjects for whom mean land slope angles could not be calculated due to the resolution of the data.

\section{Outcome variable}

Limb muscle mass was measured with a body composition analyzer using bioimpedance method (MC-780A, Tanita Corporation, Tokyo, Japan). Skeletal muscle mass index (SMI) was calculated based on limb skeletal muscle mass divided by the square of body height. Muscle function was assessed based on the maximum grip strength measured by two attempts in each hand. Higher levels of muscle mass and muscle function are related to better health [1-4]. We examined continuous changes in SMI and maximum grip strength over time. As a sensitivity analysis, we examined the number of new sarcopenia cases between 2016 and 2019. According to the genderspecific Asian sarcopenia criteria, we classified those having sarcopenia as follows: SMI in males $<7.0 \mathrm{~kg} / \mathrm{m}^{2}$ and females $<5.6 \mathrm{~kg} / \mathrm{m}^{2}$ and maximum grip strength in males $<28 \mathrm{~kg}$ and females $<18 \mathrm{~kg}$ [26]. For this analysis, we created a different subset of the cohort by excluding 


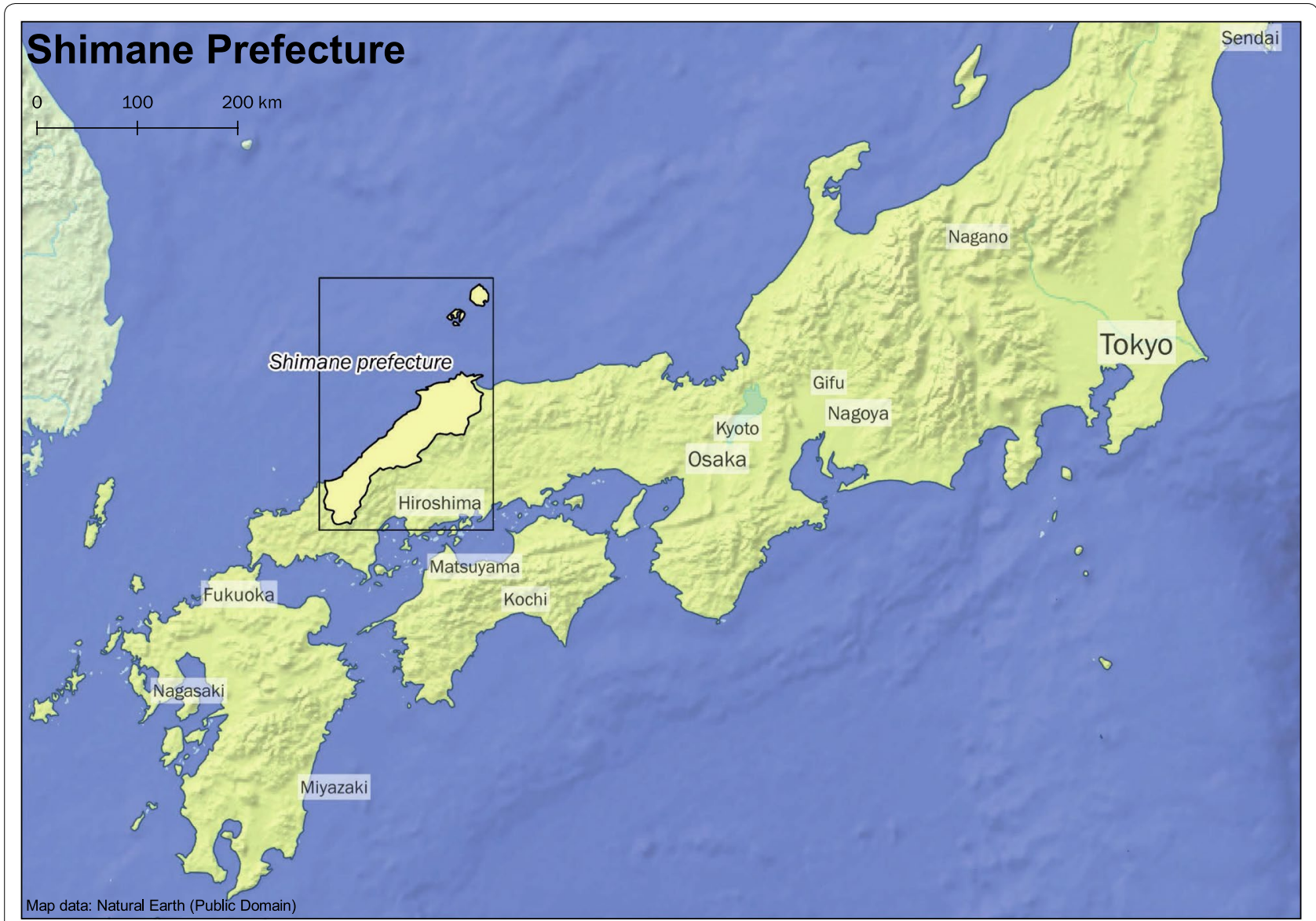

Fig. 1 Study area: Shimane Prefecture in Japan

subjects who had sarcopenia already in the first year of participation (2016, 2017, or 2018), and included those who had more than 2 measurements between 2016 and 2019.

\section{Exposure variables}

Hilliness, bus stop density, intersection density, residential density, and distance to community centers were measured by geographic information systems (GIS). The measures for hilliness, bus stop density, intersection density and residential density were calculated within a $1000 \mathrm{~m}$ network buffer from the point of residence of each subject based on actual street network. We used $1000 \mathrm{~m}$ network buffers, which has been found to be an appropriate space for activities in previous neighborhood studies [27]. Land slope was used to assess the hilliness of the neighborhood. Hilly neighborhoods have been reported to be associated with both higher and lower physical activity and weight gain in older adults [28-30]. The mean land slope was computed based on the Elevation and degree of Slope 5th Mesh Data obtained from the National Land Numerical Information (NLNI), which is publicly available GIS data administered by the National Land Information Division, National Spatial Planning, and Regional Policy Bureau of Japan. We calculated the mean land slope for each individuals' network buffer by the value of the slope stored in each $50 \mathrm{~m}$ grid (5th Mesh Data) which intersected with network buffers. Higher bus stop density, intersection density and residential density have been reported to be associated with higher physical activity levels and better health in older adults [31, 32]. Both bus stop and intersection density were calculated based on the number of points, i.e., bus stops and intersections with three or more legs within network buffers. Point data of bus stops was obtained from the Detailed Map, ArcGIS data collection, and point data of intersections was obtained from the Street Network, ArcGIS data collection administered by Esri Corporation, Tokyo, Japan (Esri Japan). Residential density was calculated using the number of households located in the smallest Japanese census statistics areas within network buffers, which was also obtained as point data from the ArcGIS data collection from Esri Japan. Distance to a community center was calculated based on 


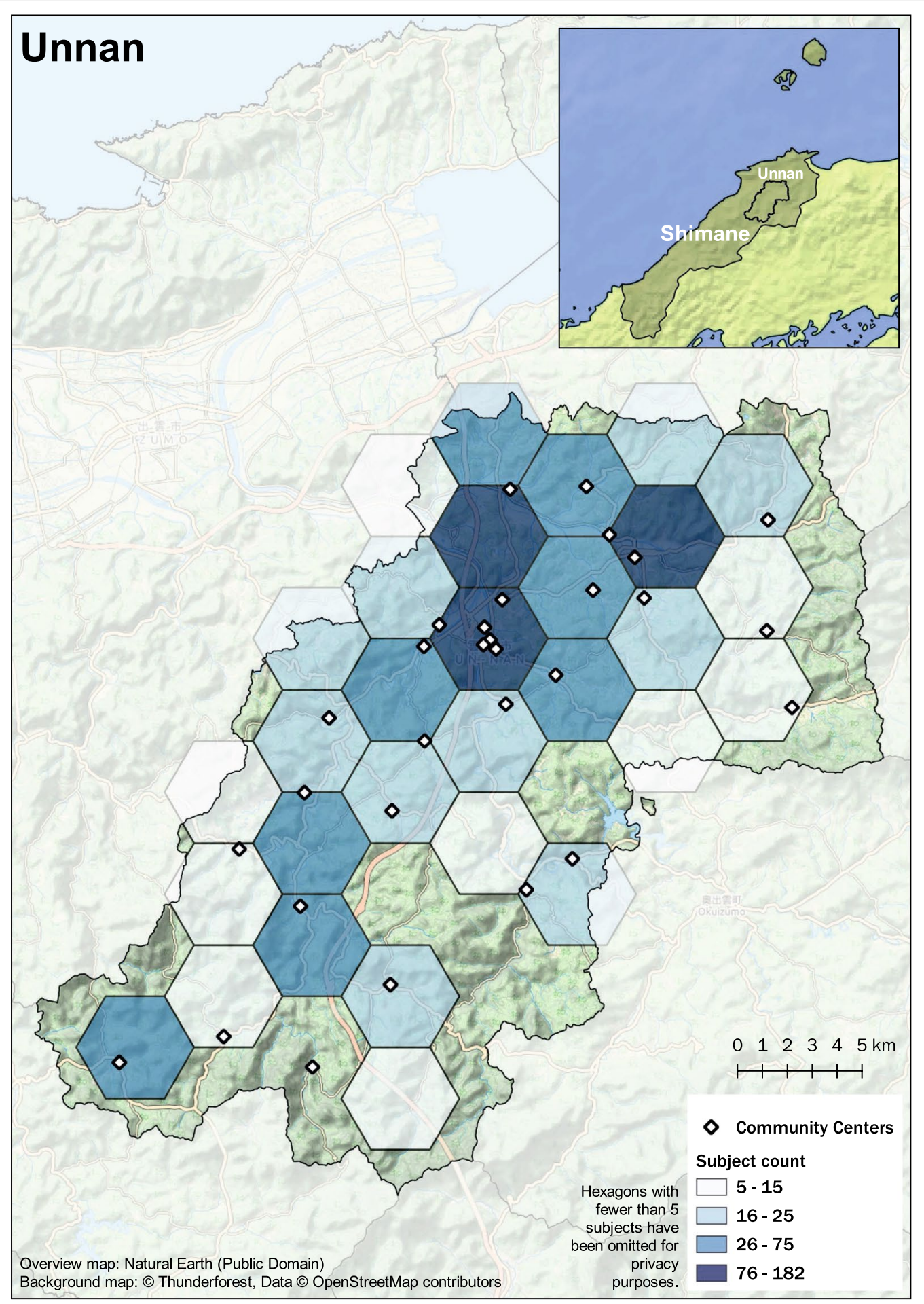

Fig. 2 Distribution of study samples in Unnan, Shimane

street networks from each point of residence to the community center of the subject's community district. Community centers in Japan are public facilities that provide educational, cultural and other recreational activities to the community. It is also a place for local associations, such as senior citizens' clubs, to organize regular 


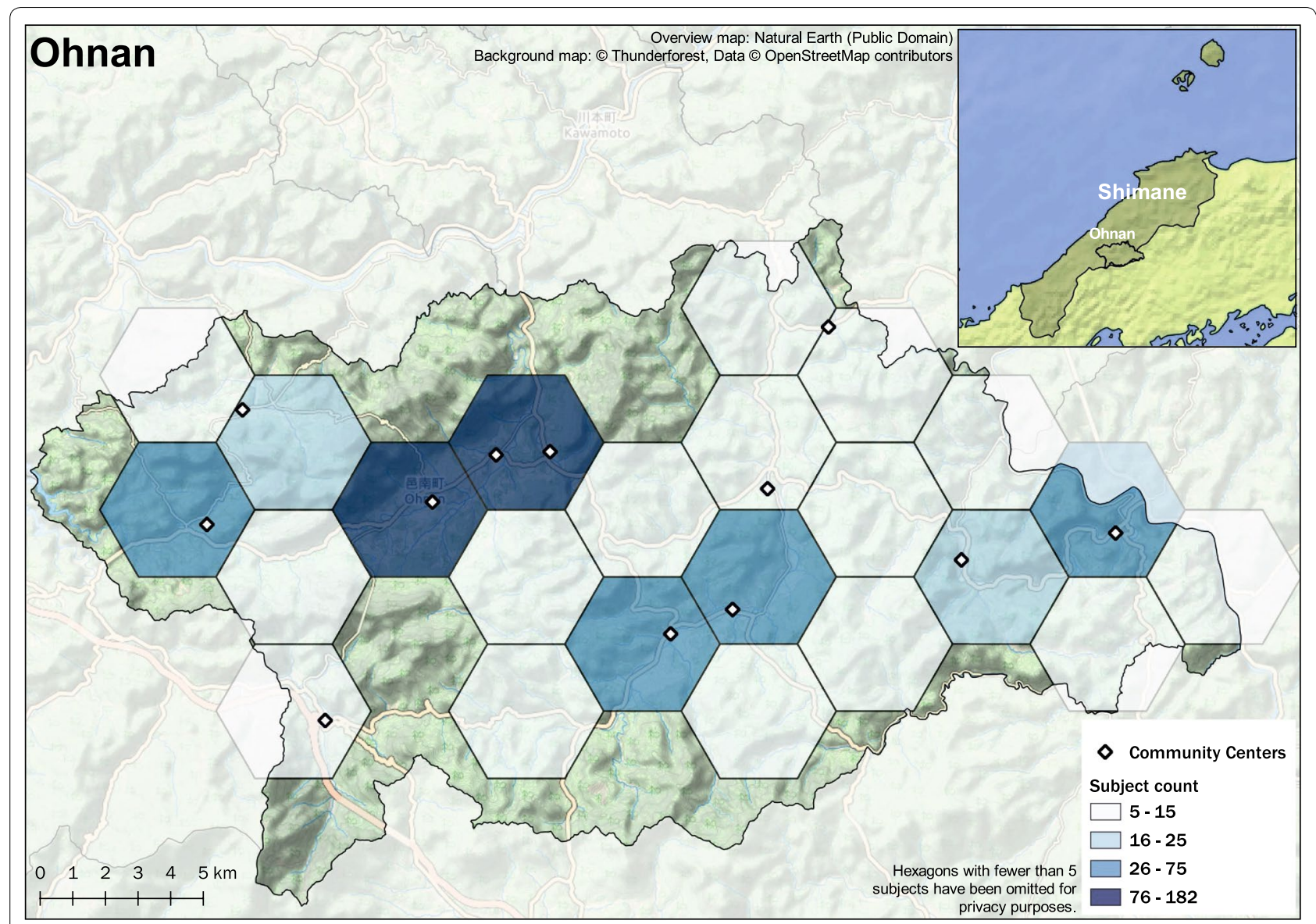

Fig. 3 Distribution of study samples in Ohnan, Shimane

meetings. Previous research has shown that participating in social activities at community centers contributes to the maintenance of activities of daily living among older adults [33]. The calculated values were categorized by using medians into two groups (high, low or close, far), and their effects on the changes in SMI and maximum grip strength over time were analyzed. All spatial analyses were done by ArcGIS Pro 2.0 (Esri Japan).

\section{Covariates}

Factors that could potentially confound or modify the association between neighborhood environmental factors and SMI and grip strength, including age, residential town, smoking, drinking, physical activity habits, musculoskeletal disorders, cardiovascular disease, and cerebrovascular disease, were obtained through selfadministered questionnaires and were included in the analysis. Lifestyle factors such as smoking, drinking and physical activity are associated with neighborhood environmental factors [31, 32], as well as risk factors of sarcopenia [3]. Chronic conditions such as musculoskeletal disorders, cardiovascular disease and cerebrovascular disease are known risk factors of sarcopenia [3]. Therefore, they were included as adjustment variables in the analyses of the association between neighborhood environmental factors and SMI and grip strength.

\section{Statistical analysis}

Frequency and proportion for the subjects' baseline characteristics are described by gender. Changes in SMI and maximum grip strength over time are reported as the mean and standard deviation by gender, age, and residential town. The influence of neighborhood environmental factors on SMI and maximum grip strength was analyzed using a linear mixed effects model. The linear mixed effects model estimates the intra-individual variation as random effects when repeated measures of individual data are available to estimate the fixed effect of the explanatory variable on the target variable over time [34]. Whether to include the intercept and slope of the individual as random effects was determined by the Akaike Information Criterion (AIC). We used a model that estimated both the intercept and the slope as a random effect for all 


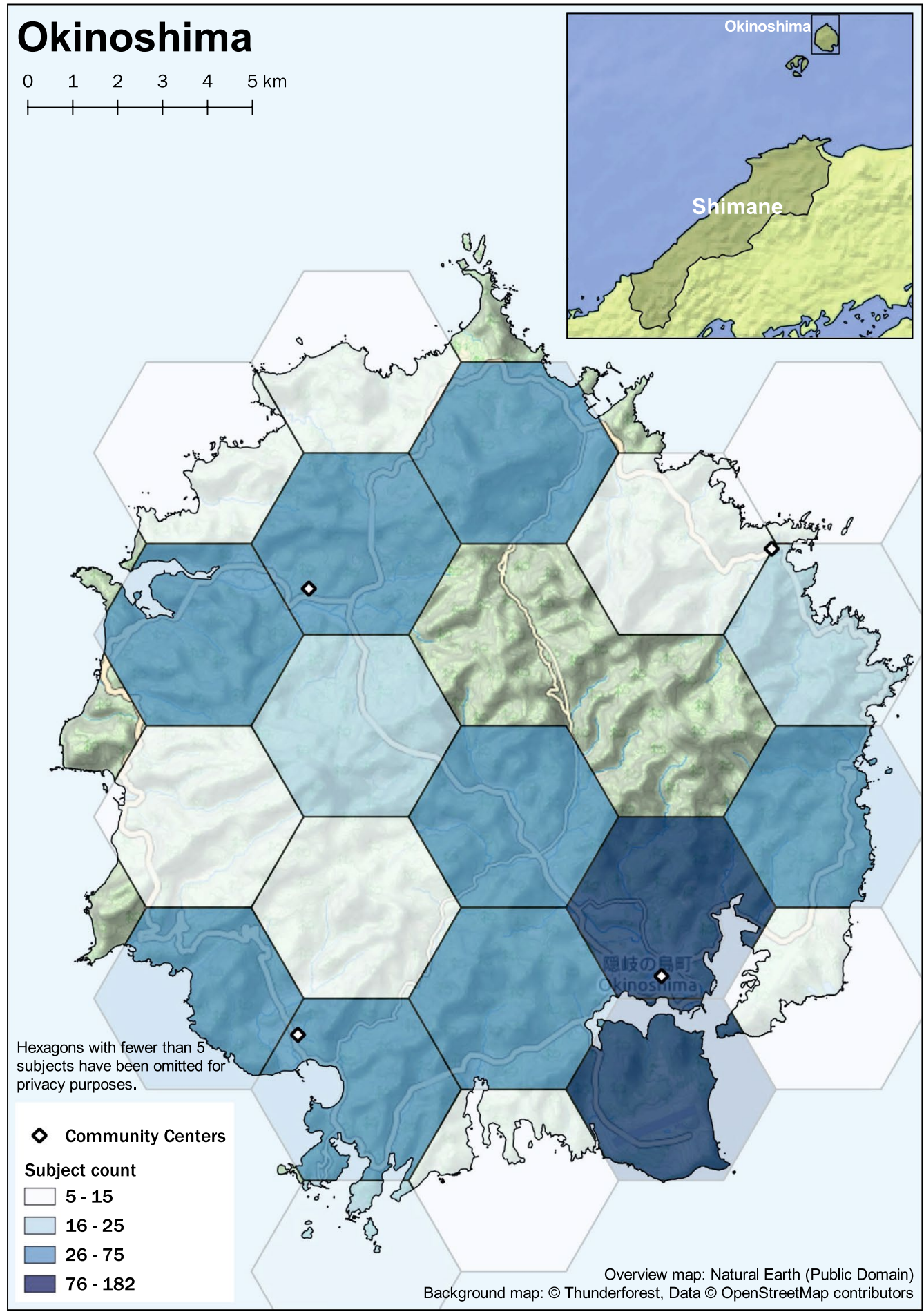

Fig. 4 Distribution of study samples in Okinoshima, Shimane

analyses. All neighborhood environmental factors were included separately in the models to avoid multicollinearity (correlation analysis was conducted and is shown in Additional file 1: Tables S1, S2). Model 1 included only time, i.e., year for assessment of change of outcomes. Model 2 also included the exposure, i.e., 
the neighborhood environmental factors, to assess the change of outcomes adjusting for all the covariates. As a sensitivity analysis, we described the number of new sarcopenia cases between 2016 and 2019. We used R 3.6.1, an open source statistical tool for the analysis, and the threshold for statistical significance was set to $<0.05$.

\section{Results}

\section{Subject characteristics}

The final analysis included 2526 subjects (1013 males and 1513 females), characterized by the factors included in the self-administered questionnaire (Table 1).

\section{Temporal changes in SMI and maximum grip strength}

In both males and females, simple aggregated means of both SMI and grip strength were higher among younger age groups, i.e., $60-75$ years old, compared to older age group, i.e., 76 years and above at all year points. Mean SMI and grip strength were both higher among males. In the respective gender and age groups, simple aggregated mean by year showed that there was no apparent change in SMI or maximum grip strength over time (Table 2).

Table 1 Characteristics of study subjects at the baseline (2016) by gender

\begin{tabular}{lll}
\hline & Male & Female \\
\hline $\mathrm{n}$ & 1013 & 1513 \\
Age $=60-75 / 76+(\%)$ & $712 / 301(70.3 / 29.7)$ & $1073 / 440(70.9 / 29.1)$ \\
Town of residence (\%) & & \\
Unnan & $492(48.6)$ & $625(41.3)$ \\
Oki & $261(25.8)$ & $512(33.8)$ \\
Onan & $260(25.7)$ & $376(24.9)$ \\
Smoking status =Yes (\%) & $159(15.7)$ & $13(0.9)$ \\
Drinking status (\%) & & $133(8.8)$ \\
Yes & $532(52.5)$ & $280(18.5)$ \\
Occasionally & $183(18.1)$ & $1100(72.7)$ \\
No & $298(29.4)$ & $760(50.2)$ \\
Physical activity habit=Yes (\%) & $524(51.7)$ & $486(32.1)$ \\
Musculoskeletal disorders $=$ Yes (\%) & $153(15.1)$ & $114(7.5)$ \\
Cardiovascular disease history $=$ Yes (\%) & $109(10.8)$ & $45(3.0)$ \\
Cerebrovascular disease history=Yes (\%) & $65(6.4)$ & \\
\hline
\end{tabular}

Table 2 Mean SMI and grip strength across 4 waves by age and towns

\begin{tabular}{|c|c|c|c|c|c|c|c|c|c|}
\hline & & \multicolumn{4}{|c|}{ SMI, mean (sd) } & \multicolumn{4}{|c|}{ Grip strength, mean (sd) } \\
\hline & & 2016 & 2017 & 2018 & 2019 & 2016 & 2017 & 2018 & 2019 \\
\hline \multicolumn{10}{|l|}{ Male } \\
\hline \multirow[t]{2}{*}{ Unnan } & $60-75$ & $7.6(0.9)$ & $7.7(0.9)$ & $7.6(0.8)$ & $7.6(0.9)$ & $37.2(5.7)$ & $37.3(5.8)$ & $37.1(5.9)$ & $37.1(5.1)$ \\
\hline & $76+$ & $7.4(0.8)$ & $7.2(0.7)$ & $7.3(0.8)$ & $7.2(0.8)$ & $32.4(5.8)$ & $32.8(5.7)$ & $33.5(4.4)$ & $33.6(4.8)$ \\
\hline \multirow[t]{2}{*}{ Oki } & $60-75$ & $7.8(1)$ & $7.8(1)$ & $7.7(0.9)$ & $7.8(0.9)$ & $38.9(6.2)$ & $37.6(5.7)$ & $37(5.9)$ & $38.7(6.5)$ \\
\hline & $76+$ & $7.5(0.8)$ & $7.4(0.9)$ & $7.4(0.9)$ & $7.4(0.9)$ & $33.8(5.7)$ & $33.4(5.2)$ & $32(5.5)$ & $32.9(5.7)$ \\
\hline Onan & $60-75$ & $7.8(1)$ & $7.9(1.1)$ & & $7.7(1)$ & $37.2(5.4)$ & $36.6(5.5)$ & & $36.4(5.5)$ \\
\hline \multicolumn{10}{|l|}{ Female } \\
\hline \multirow[t]{2}{*}{ Unnan } & $60-75$ & $6.2(0.7)$ & $6.2(0.7)$ & $6.2(0.7)$ & $6.2(0.7)$ & $23.7(3.7)$ & $25.3(3.7)$ & $24.3(3.9)$ & $24.1(3.4)$ \\
\hline & $76+$ & $6.1(0.8)$ & $5.9(0.6)$ & $5.9(0.6)$ & $5.9(0.8)$ & $21(3.8)$ & $22.2(4.1)$ & $21.9(3.9)$ & $22(3.8)$ \\
\hline \multirow[t]{2}{*}{ Oki } & $60-75$ & $6.4(0.7)$ & $6.3(0.6)$ & $6.3(0.7)$ & $6.4(0.8)$ & $24.4(3.6)$ & $23.5(3.5)$ & $23.9(3.7)$ & $23.9(3.8)$ \\
\hline & $76+$ & $6.2(0.8)$ & $6.1(0.8)$ & $6.1(0.8)$ & $6(0.6)$ & $21(3.7)$ & 20.5 (3.6) & $21.2(4.1)$ & $20.9(3.7)$ \\
\hline Onan & $60-75$ & $6.4(0.7)$ & $6.3(0.7)$ & & $6.4(0.8)$ & $23.8(3.9)$ & $24.3(4.2)$ & & $23.6(3.8)$ \\
\hline
\end{tabular}




\section{Association between neighborhood environment factors, $\mathrm{SMI}$, and grip strength}

In males, the linear mixed model using only the year (time) showed that both SMI and maximum grip strength decreased significantly each year $(\beta:-0.05,95 \%$ $\mathrm{CI}-0.06,-0.04 \quad$ (SMI); $\beta:-0.41,95 \% \mathrm{CI}-0.49,-0.32$ (maximum grip strength)) (Table 3). The model, including neighborhood environmental factors and potential confounding factors, showed that SMI was significantly lower in areas with a higher density of bus stops $(\beta$ $:-0.15,95 \% \mathrm{CI}-0.27,-0.03)$. This was interpreted as the average effect of bus stop density over time, i.e., assessed at 4 time points from 2016 to 2019. In other words, the coefficient -0.15 is the difference in SMI between the area with high bus stop density and low bus stop density in average based on the 4 time points. The purpose was to examine how the changes (trajectories) differ in 2017, 2018, and 2019. The change in mean SMI over time was only associated with distance to community center $(\beta: 0.04,95 \%$ CI $0.01,0.07)$, which indicated that living far from a community center would alleviate a decline in SMI. With respect to grip strength, an average effect was only found for land slope, i.e., grip strength was significantly higher in areas with a higher land slope ( $\beta: 0.93,95 \%$ CI 0.18, 1.68). No significant differences in the change of grip strength over time were found for any of the other environmental factors.

In females, like males, the model including the year only showed that both SMI and maximum grip strength decreased significantly each year $(\beta:-0.03,95 \%$
$\mathrm{CI}-0.03,-0.02$ (SMI); $\beta:-0.21,95 \% \mathrm{CI}-0.26,-0.16$ (maximum grip strength)) (Table 4). Neighborhood environmental factors that significantly affected the changes of SMI were not identified. With respect to grip strength, an average effect was found for bus stop density and distance to a community center, i.e., it was significantly lower in areas with a higher density of bus stops ( $\beta$ $:-0.61,95 \% \mathrm{CI}-1.03,-0.18)$ and significantly higher in areas far away from a community center $(\beta: 0.53,95 \% \mathrm{CI}$ $0.11,0.96)$. The changes in mean grip strength over time was only associated with residential density ( $\beta: 0.13,95 \%$ CI 0.02, 0.24), which indicated that living in areas with high residential density would alleviate a decline in grip strength.

\section{Number of new sarcopenia cases}

Few new cases of sarcopenia (6 in males and 11 in females) were identified between 2016 and 2019 (Table 5).

\section{Discussion}

The results of this study showed that older men living in areas far from community centers and women living in areas with high residential density had a less pronounced decline in SMI and grip strength over time compared to their counterparts. The effects of neighborhood environmental factors on both older men and women appeared small, yet this may have been due to the relatively short follow-up of 3 years; it is possible that a short follow-up only carries a subtle change of muscle mass and function

Table 3 Male-linear mixed model for SMI and grip strength by environment variable

\begin{tabular}{|c|c|c|c|c|}
\hline & \multicolumn{2}{|l|}{ SMI } & \multicolumn{2}{|l|}{ Grip strength } \\
\hline & Model $1^{\mathrm{a}}$ & Model $2^{b}$ & Model $1^{\mathrm{a}}$ & Model $2^{b}$ \\
\hline Variables & $\beta(95 \% \mathrm{Cl})$ & $\beta(95 \% \mathrm{Cl})$ & $\beta(95 \% \mathrm{Cl})$ & $\beta(95 \% \mathrm{Cl})$ \\
\hline Year & $-0.05^{*}(-0.06,-0.04)$ & - & $-0.41^{*}(-0.49,-0.32)$ & - \\
\hline Land slope $(r e f=$ Low $)$ & & $0.01(-0.11,0.14)$ & & $0.93^{*}(0.18,1.68)$ \\
\hline Year*Land slope $(r e f=$ Low $)$ & & $0(-0.03,0.03)$ & & $-0.01(-0.2,0.18)$ \\
\hline Bus stop density (ref $=$ Low) & & $-0.15^{*}(-0.27,-0.03)$ & & $-0.33(-1.09,0.43)$ \\
\hline Year*Bus stop density (ref $=$ Low) & & $0.01(-0.02,0.04)$ & & $-0.07(-0.26,0.12)$ \\
\hline Intersection density (ref $=$ Low) & & $0.01(-0.11,0.14)$ & & $-0.49(-1.24,0.26)$ \\
\hline Year*Intersection density (ref $=$ Low) & & $0(-0.03,0.03)$ & & $0.13(-0.06,0.31)$ \\
\hline Residential density (ref = Low) & & $-0.05(-0.17,0.07)$ & & $-0.13(-0.89,0.62)$ \\
\hline Year*Residential density $(r e f=L o w)$ & & $-0.01(-0.04,0.02)$ & & $0.03(-0.16,0.21)$ \\
\hline Distance to community center (ref $=$ Close) & & $-0.03(-0.15,0.1)$ & & $0.37(-0.39,1.13)$ \\
\hline Year*Distance to community center (ref $=$ Close) & & $0.04^{*}(0.01,0.07)$ & & $-0.02(-0.21,0.17)$ \\
\hline
\end{tabular}

Environment factors were added separately into all models

${ }^{*} \mathrm{p}<0.05$

${ }^{a}$ Model 1: time only model

b Model 2: conditional growth model by each environment factor adjusting for all covariates, i.e. age, town of residence, smoke, drink, physical activity, musculoskeletal disorders, cardiovascular disease, cerebrovascular diseases 
Table 4 Female-linear mixed model for SMI and grip strength by environment variable

\begin{tabular}{|c|c|c|c|c|}
\hline & \multicolumn{2}{|l|}{ SMI } & \multicolumn{2}{|l|}{ Grip strength } \\
\hline & Model $1^{\mathrm{a}}$ & Model $2^{\text {b }}$ & Model $1^{\mathrm{a}}$ & Model $2^{b}$ \\
\hline Variables & $\beta(95 \% \mathrm{Cl})$ & $\beta(95 \% \mathrm{Cl})$ & $\beta(95 \% \mathrm{Cl})$ & $\beta(95 \% \mathrm{Cl})$ \\
\hline Year & $-0.03^{*}(-0.03,-0.02)$ & - & $-0.21^{*}(-0.26,-0.16)$ & $-0.1(-0.18,-0.02)$ \\
\hline Land slope $($ ref $=$ Low $)$ & & $-0.01(-0.09,0.07)$ & & $0.09(-0.33,0.51)$ \\
\hline Year*Land slope $(r e f=$ Low $)$ & & $0(-0.02,0.02)$ & & $-0.05(-0.16,0.06)$ \\
\hline Bus stop density (ref $=$ Low) & & $-0.06(-0.14,0.02)$ & & $-0.61^{*}(-1.03,-0.18)$ \\
\hline Year*Bus stop density (ref $=$ Low) & & $0.01(-0.01,0.04)$ & & $0.07(-0.04,0.18)$ \\
\hline Intersection density (ref $=$ Low $)$ & & $-0.03(-0.11,0.05)$ & & $-0.3(-0.72,0.12)$ \\
\hline Year*|ntersection density (ref $=$ Low) & & $0.01(-0.02,0.03)$ & & $0.01(-0.1,0.12)$ \\
\hline Residential density (ref = Low) & & $-0.02(-0.1,0.05)$ & & $-0.26(-0.69,0.16)$ \\
\hline Year*Residential density $(r e f=$ Low $)$ & & $0.01(-0.02,0.03)$ & & $0.13^{*}(0.02,0.24)$ \\
\hline Distance to community center (ref $=$ Close) & & $0.02(-0.06,0.1)$ & & $0.53^{*}(0.11,0.96)$ \\
\hline Year*Distance to community center (ref $=$ Close) & & $-0.01(-0.03,0.01)$ & & $-0.05(-0.16,0.06)$ \\
\hline
\end{tabular}

Environment factors were added separately into all models

* $\mathrm{p}<0.05$

${ }^{a}$ Model 1: time only model

${ }^{b}$ Model 2: conditional growth model by each environment factor adjusting for all covariates, i.e. age, town of residence, smoke, drink, physical activity, musculoskeletal disorders, cardiovascular disease, cerebrovascular diseases

Table 5 Number of incidence of sarcopenia between 2016 and 2019 by gender

\begin{tabular}{lll}
\hline & Male & Female \\
\hline $\mathrm{n}$ & 775 & 1140 \\
$\begin{array}{l}\text { Sarcopenia= No/Yes } \\
(\%)\end{array}$ & $769 / 6(99.2 / 0.8)$ & $1129 / 11(99.0 / 1.0)$ \\
\hline
\end{tabular}

in older adults over 60 years, which is supported by the low incidence of sarcopenia. It has been reported that decline in physical function and increased risk of sarcopenia in older adult populations requires a longer period to occur [35]. Additionally, subjects in this study had voluntarily opted to participate in group health examinations and they may therefore have been more conscious of their health and physically active. It was nonetheless shown that certain neighborhood environmental factors might affect longitudinal change of muscle mass and function for older men and women.

Previous studies have found that high social cohesion was associated with lower functional limitations $[11,14]$. Living close to community centers can be hypothesized to increase the likelihood of engaging in social activities organized by community centers, and thus leading to increased social cohesion. However, our findings showed that living close to community centers had potentially detrimental effects on the decline in SMI among older men. Potential reasons for this are, firstly, living physically close to community centers does not necessarily mean participating in social activities or belonging to active groups. While it has been reported that proximity to resources was associated with social participation [36], whether or not individuals participated in these group activities should be assessed separately. Secondly, social norms within a tightly-knit group have been reported to be both health-promoting and health-damaging to older adults. Older men living physically close to community centers might be under more social pressure to get involved in activities, and that could lead to psychological stress and could negatively affect health because older men in general may be less willing to participate in social activities than older women [36]. Although further studies are needed to suggest policy implications, it may be possible to modify the types and/or frequency of activities held within community centers to be more beneficial to the residents. Thirdly, in our rural study areas, there are also smaller hubs besides community centers that organized social activities and it was not possible to capture these in our data. This might have biased our results in an unexpected direction.

High residential density has been found to contribute to the promotion of physical activity and improve certain health outcomes in older adults, which is consistent with our findings for older women [31, 32]. Residential density might be a more important health-promoting factor in women than in men, especially in rural settings. Moreover, it is less common for women to have a driving license than men; this is even more evident among older populations in rural parts of Japan. A previous study showed that more physical activity was observed 
among drivers than non-drivers as they were assumed to get to destinations where they can engage in exercise [37]. Another study showed that public transit access was more important to non-drivers than drivers among rural Japanese women [38]. However, the mechanisms of such associations were not examined in this study, and there is insufficient evidence to provide suggestions on policy. In addition, because it is difficult to change the residential density of an area, it may be more important to focus on other modifiable environmental factors, such as locations of commercial facilities. Commercial facilities, such as food stores and physical activity facilities (e.g. sports gym), are potentially modifiable via regulations in a relatively short time span compared to street or city layout, which require significant investment over long time periods. Several previous studies have examined these commercial facilities and found that they are associated with older adults' nutritional status and physical activity level $[39,40]$.

This study has several limitations. First, the study subjects were not randomly sampled from each residential town. Our study subjects were limited to those who participated in the annual health examinations; thus, they might have been more conscious of their health than the general older adult population. This might explain why only subtle changes in SMI and grip strength as well as a low incidence of sarcopenia was observed in this study, in addition to a short follow-up period. Second, our study did not include aspects of individual and neighborhood factors relating to diet, an important determinant of muscle mass and strength in older adults [7]. Although the effect of diet on sarcopenia is not likely to be as firm as physical activity [24, 41], it should be investigated at the environmental level, such as locations of grocery and convenience stores, where intervention could possibly be implemented.

This study also has several strengths. First, this is the first study that has examined the potential effect of the neighborhood environment on muscle mass and function in more than 2000 older adults ( $\geq 60$ years old) as well as in males and females separately, using a longitudinal study design. Most previous studies on neighborhood environments and physical function included middle age adults, such as 40 or 50 years old together with older adults and most studies were cross-sectional [10-15, 42-45]. Second, our study objectively measured both neighborhood environmental factors as well as muscle mass and function specific to sarcopenia. Although subjective measures are also important, they vary between individuals [46] and using objective measures is therefore more helpful to design a meaningful intervention. Third, our study targeted rural areas of Japan where the population aged more than 65 was already exceeding $35 \%$.
Despite the fact that rural populations are at a higher risk of chronic health conditions in general due to limited resources available $[47,48]$, studies targeting rural populations are limited [31, 32]. It is therefore crucial to study rural populations as a higher percentage of aging people are often found in rural settings, and this is likely to increase further in several countries across the world [49].

The Japanese government aims to establish community-based integrated care systems to enable elderly people to have an independent life in their own neighborhoods by 2025 [50]. Community centers play an important role for community-based services to elderly people and the findings of this study could thus be utilized when interventions are planned for rural elderly residents who often have difficulties in terms of access to many facilities and activities. Local governments may take measures to improve both the access and use of such facilities and activities based on objectively measured numerical indicators. In our study, we found that older men living far from community centers $(\geq 1640 \mathrm{~m})$ maintained their SMI better than those living close to community centers $(<1640 \mathrm{~m})$. We also found that older women living in areas with more households $(\geq 45)$ within a $1000 \mathrm{~m}$ network buffer maintained their grip strength better than those living in areas with fewer households $(<45)$ although an optimal threshold of residential density that can be beneficial for good health is unclear [51].

The findings of this study may suggest local governments, such as that in Shimane prefecture, to take a closer look on the specific types of facilities and activities offered at community centers and their potential effects on elderly people's health rather than merely improving the proximity to community centers. Given the potential effects of the use of community centers for physical function improvements among older adults [33], it is, however, useful to know which areas and populations that need more focused interventions based on objectively collected data.

\section{Conclusions}

Although the objectively measured neighborhood environmental factors in rural areas mostly had a quite limited effect on objectively measured muscle mass and muscle function decline in older adults there were certain neighborhood features that affected these outcomes more and some gender differences also appeared. This calls for further studies in other countries in order to investigate other modifiable environmental factors as well as following elderly individuals over a longer period. 


\section{Supplementary information}

Supplementary information accompanies this paper at https://doi. org/10.1186/s12942-020-00247-9.

Additional file 1: Table S1. Correlation between neighborhood environment factors among males. Table S2. Correlation between neighborhood environment factors among females.

\section{Acknowledgements}

We would like to express our sincere gratitude to the residents who have invested a great deal of time and effort into this research, the officials of municipalities who have jointly conducted the survey with our center for more than 10 years, and the staff of our center who worked to implement the survey and organize the data. Finally, we would like to thank the Meiji Yasuda Life Foundation of Health and Welfare for supporting this research as well as the Swedish Heart-Lung foundation to Kristina Sundquist.

\section{Authors' contributions}

$\mathrm{KO}$ designed the study, prepared and analyzed the data, and was a major contributor in writing this manuscript. TA contributed conception and design of the work, as well as analysis and data interpretation before and throughout the study, and revisions of manuscript. SY contributed the acquisition of longitudinal data, and also contributed the conception of the article in the beginning of the study. KS and TN contributed to the conception of the study substantially in the beginning of the study, and revisions of manuscript. TN contributed the conception of the study, data acquisition, as well as analysis and interpretation of the analysis results. All authors read and approved the final manuscript.

\section{Funding}

Open Access funding provided by Lund University. This work was supported by Young Research Professionals Grant from the Meiji Yasuda Life Foundation of Health and Welfare, Tokyo Japan.

\section{Availability of data and materials}

The data used in this paper include personal residential information as well as health related information. Therefore, the data are not publicly available due to privacy concerns. The data could be requested and available from the corresponding author on safe manner in accordance with the ethical policy statements of this study protocol.

\section{Ethics approval and consent to participate}

The study protocol and procedure used to secure the informed consent of the participants were approved by the Ethics Committee of Shimane University \#2888

\section{Consent for publication}

The authors provide full consent for publishing the manuscript.

\section{Competing interests}

The authors declare that the funding source did not influence the study design, data collection, data analysis, interpretation, or reporting of this study.

\section{Author details}

${ }^{1}$ Center for Primary Health Care Research, Lund University, Jan Waldenströms gata 35, 20502 Malmö, Sweden. ${ }^{2}$ Center for Community-Based Healthcare Research and Education (CoHRE), Organization for Research and Academic Information, Shimane University, 223-8 Enya-cho, Izumo-shi, Shimane 693-8501, Japan. ${ }^{3}$ Department of Laboratory Medicine, Faculty of Medicine, Shimane University, 89-1 Enya-cho, Izumo-shi, Shimane 693-8501, Japan. ${ }^{4}$ Department of Family Medicine and Community Health, Department of Population Health Science and Policy, Icahn School of Medicine At Mount Sinai, 1 Gustave L. Levy Place, New York, NY 10029-5674, USA. ${ }^{5}$ Department of Functional Pathology, Faculty of Medicine, Shimane University, 89-1 Enya-cho, Izumo-shi, Shimane 693-8501, Japan.

Received: 8 July 2020 Accepted: 16 November 2020 Published online: 25 November 2020
References

1. Beaudart C, Zaaria M, Pasleau F, Reginster J-Y, Bruyère $O$. Health outcomes of sarcopenia: a systematic review and meta-analysis. PLoS ONE. 2017;12(1):e0169548.

2. Cruz-Jentoft AJ, Baeyens JP, Bauer JM, Boirie Y, Cederholm T, Landi F, et al. Sarcopenia: European consensus on definition and diagnosis: report of the European Working Group on Sarcopenia in Older People. Age Ageing 2010;39(4):412-23.

3. Cruz-Jentoft AJ, Bahat G, Bauer J, Boirie Y, Bruyère O, Cederholm T, et al . Sarcopenia: revised European consensus on definition and diagnosis. Age Ageing. 2019;48(1):16-31.

4. Tsekoura M, Kastrinis A, Katsoulaki M, Billis E, Gliatis J. Sarcopenia and Its Impact on Quality of Life. In: Vlamos P, editor. GeNeDis 2016. Cham: Springer International Publishing; 2017. p. 213-8.

5. Kortebein P, Ferrando A, Lombeida J, Wolfe R, Evans WJ. Effect of 10 days of bed rest on skeletal muscle in healthy older adults. JAMA. 2007;297(16):1769-74.

6. Renoud A, Ecochard R, Marchand F, Chapurlat R, Szulc P. Predictive parameters of accelerated muscle loss in men-MINOS study. Am J Med. 2014:127(6):554-61.

7. Rizzoli R, Stevenson JC, Bauer JM, van Loon LJC, Walrand S, Kanis JA, et al. The role of dietary protein and vitamin D in maintaining musculoskeletal health in postmenopausal women: a consensus statement from the European Society for Clinical and Economic Aspects of Osteoporosis and Osteoarthritis (ESCEO). Maturitas. 2014;79(1):122-32.

8. Zampieri S, Pietrangelo L, Loefler S, Fruhmann H, Vogelauer M, Burggraf $\mathrm{S}$, et al. Lifelong physical exercise delays age-associated skeletal muscle decline. J Gerontol A Biol Sci Med Sci. 2015;70(2):163-73.

9. Duncan DT, Kawachi I. Neighborhoods and health: a progress report. Oxford: Oxford University Press; 2018. https://www.oxfordscholarship. com/view/https://doi.org/10.1093/oso/9780190843496.001.0001/oso9780190843496-chapter-1.

10. Beard JR, Blaney S, Cerda M, Frye V, Lovasi GS, Ompad D, et al. Neighborhood characteristics and disability in older adults. J Gerontol B Psychol Sci Soc Sci. 2009:64B(2):252-7.

11. Feldman PJ, Steptoe A. How neighborhoods and physical functioning are related: the roles of neighborhood socioeconomic status, perceived neighborhood strain, and individual health risk factors. Ann Behav Med. 2004;27(2):91-9.

12. Loh VHY, Rachele JN, Brown WJ, Ghani F, Turrell G. Neighborhood disadvantage and physical function: the contributions of neighborhood-level perceptions of safety from crime and walking for recreation. J Phys Act Health. 2019;15(8):553-63.

13. Millar RJ. Neighborhood cohesion, disorder, and physical function in older adults: an examination of racial/ethnic differences. J Aging Health. 2019. https://doi.org/10.1177/0898264319890944

14. Nguyen TT, Rist PM, Glymour MM. Are self-reported neighbourhood characteristics associated with onset of functional limitations in older adults with or without memory impairment? J Epidemiol Community Health. 2016;70(10):1017-23.

15. Loh VHY, Rachele JN, Brown WJ, Washington S, Turrell G. Neighborhood disadvantage, individual-level socioeconomic position and physical function: a cross-sectional multilevel analysis. Preventive. 2016;89:112-20.

16. Anker SD, Morley JE, von Haehling S. Welcome to the ICD-10 code for sarcopenia. J Cachexia Sarcopenia Muscle. 2016;7(5):512-4.

17. Cooper R, Kuh D, Hardy R, Group MR. Objectively measured physical capability levels and mortality: systematic review and meta-analysis. BMJ. 2010;341:21.

18. Gale CR, Martyn CN, Cooper C, Sayer AA. Grip strength, body composition, and mortality. Int J Epidemiol. 2007;36(1):228-35.

19. Newman AB, Kupelian V, Visser M, Simonsick EM, Goodpaster BH, Kritchevsky SB, et al. Strength, but not muscle mass, is associated with mortality in the health, aging and body composition study cohort. J Gerontol A Biol Sci Med Sci. 2006:61(1):72-7.

20. Shimada H, Makizako H, Doi T, Tsutsumimoto K, Suzuki T. Incidence of disability in frail older persons with or without slow walking speed. J Am Med Direct Assoc. 2015;16(8):690-6.

21. Shinkai S, Watanabe S, Kumagai S, Fujiwara Y, Amano H, Yoshida H, et al. Walking speed as a good predictor for the onset of functional dependence in a Japanese rural community population. Age Ageing. 2000;29(5):441-6. 
22. Syddall H, Cooper C, Martin F, Briggs R, Aihie SA. Is grip strength a useful single marker of frailty? Age Ageing. 2003;32(6):650-6.

23. Bruyère $\mathrm{O}$, Beaudart $\mathrm{C}$, Ethgen $\mathrm{O}$, Reginster $J-Y$, Locquet $M$. The health economics burden of sarcopenia: a systematic review. Maturitas. 2019;119:61-9.

24. Cruz-Jentoft AJ, Landi F, Schneider SM, Zúñiga C, Arai H, Boirie Y, et al. Prevalence of and interventions for sarcopenia in ageing adults: a systematic review. Report of the International Sarcopenia Initiative (EWGSOP and IWGS). Age Ageing. 2014;43(6):748-59.

25. Rachele JN, Sugiyama T, Davies S, Loh VHY, Turrell G, Carver A, et al. Neighbourhood built environment and physical function among mid-to-older aged adults: a systematic review. Health Place. 2019;58:102137.

26. Chen LK, Woo J, Assantachai P, Auyeung TW, Chou MY, lijima K, et al. Asian Working Group for Sarcopenia: 2019 consensus update on sarcopenia diagnosis and treatment. J Am Med Direct Assoc 2020. doi: https://doi. org/10.1016/j.jamda.2019.12.012

27. Mavoa S, Bagheri N, Koohsari MJ, Kaczynski AT, Lamb KE, Oka K, et al. How do neighbourhood definitions influence the associations between built environment and physical activity? Int J Environ Res Public Health. 2019:16(9):1501.

28. Hanibuchi T, Kawachi I, Nakaya T, Hirai H, Kondo K. Neighborhood built environment and physical activity of Japanese older adults: results from the Aichi Gerontological Evaluation Study (AGES). BMC Public Health. 2011:11(1):657.

29. Okuyama K, Abe T, Hamano T, Takeda M, Sundquist K, Sundquist J, et al. Hilly neighborhoods are associated with increased risk of weight gain among older adults in rural Japan: a 3-years follow-up study. Int J Health Geogr. 2019;18(1):10.

30. Abe T, Okuyama K, Hamano T, Takeda M, Isomura M, Nabika T. Hilly environment and physical activity among community-dwelling older adults in Japan: a cross-sectional study. BMJ Open. 2020;10(3):e033338.

31. Barnett DW, Barnett A, Nathan A, Van Cauwenberg J, Cerin E, on behalf of the Council on Environment and Physical Activity (CEPA)_-Older Adults working group. Built environmental correlates of older adults' total physical activity and walking: a systematic review and meta-analysis. Int J Behav Nutr Phys Act 2017;14(1):103.

32. Cerin E, Nathan A, van Cauwenberg J, Barnett DW, Barnett A, Council on Environment and Physical Activity (CEPA)_Older Adults working group. The neighbourhood physical environment and active travel in older adults: a systematic review and meta-analysis. Int J Behav Nutr Phys Act. 2017;14(1):15

33. Hosokawa R, Kondo K, Ito M, Miyaguni Y, Mizutani S, Goto F, et al. The effectiveness of Japan's Community Centers in facilitating social participation and maintaining the functional capacity of older people: research on aging. 2018. https://journals.sagepub.com/doi/https://doi. org/10.1177/0164027518805918?url_ver=Z39.88-2003\&rfr_id=ori\%3Arid \%3Acrossref.org\&rfr_dat=cr_pub++0pubmed

34. Singer JD, Willett JB. Applied longitudinal data analysis: modeling change and event occurrence. Oxford: Oxford University Press; 2003. https://www.oxfordscholarship.com/view/https://doi.org/10.1093/acpro f:oso/9780195152968.001.0001/acprof-9780195152968.

35. Granic A, Davies K, Jagger C, Kirkwood TBL, Syddall HE, Sayer AA. Grip strength decline and its determinants in the very old: longitudinal findings from the newcastle 85+ study. PLoS ONE. 2016;11:9.

36. Levasseur M, Généreux M, Bruneau J-F, Vanasse A, Chabot É, Beaulac C, et al. Importance of proximity to resources, social support, transportation and neighborhood security for mobility and social participation in older adults: results from a scoping study. BMC Public Health. 2015;15(1):503.
37. Amagasa S, Fukushima N, Kikuchi H, Takamiya T, Odagiri Y, Oka K, et al. Drivers are more physically active than non-drivers in older adults. Int J Environ Res Public Health. 2018;15(6):28.

38. Kamada M, Kitayuguchi J, Inoue S, Kamioka H, Mutoh Y, Shiwaku K. Environmental correlates of physical activity in driving and non-driving rural Japanese women. Prev Med. 2009;49(6):490-6.

39. Nakamura H, Nakamura M, Okada E, Ojima T, Kondo K. Association of food access and neighbor relationships with diet and underweight among community-dwelling older Japanese. J Epidemiol. 2017;27(11):546-51.

40. Petrella RJ, Kennedy E, Overend TJ. Geographic determinants of healthy lifestyle change in a community-based exercise prescription delivered in family practice. Environ Health Insights. 2008. https://journals.sagepub. com/doi/https://doi.org/10.4137/ehi.s820?url_ver=Z39.88-2003\&rfr_ $i d=$ ori\%3Arid\%3Acrossref.org\&rfr_dat=cr_pub++0pubmed

41. Denison HJ, Cooper C, Sayer AA, Robinson SM. Prevention and optimal management of sarcopenia: a review of combined exercise and nutrition interventions to improve muscle outcomes in older people. Clin Interv Aging. 2015;10:859-69.

42. Freedman VA, Grafova IB, Schoeni RF, Rogowski J. Neighborhoods and disability in later life. Soc Sci Med. 2008;66(11):2253-67.

43. Rosso AL, Auchincloss AH, Michael YL. The urban built environment and mobility in older adults: a comprehensive review. J Aging Res. 2011;2011:e816106

44. Wight RG, Cummings JR, Miller-Martinez D, Karlamangla AS, Seeman TE, Aneshensel CS. A multilevel analysis of urban neighborhood socioeconomic disadvantage and health in late life. Soc Sci Med. 2008;66(4):862-72.

45. Yen $I H$, Michael $Y L$, Perdue L. Neighborhood environment in studies of health of older adults: a systematic review. Am J Prevent Med. 2009;37(5):455-63.

46. Godhwani S, Jivraj S, Marshall A, Bécares L. Comparing subjective and objective neighbourhood deprivation and their association with health over time among older adults in England. Health Place. 2019;55:51-8.

47. Eberhardt MS, Pamuk ER. The importance of place of residence: examining health in rural and nonrural areas. Am J Public Health. 2004;94(10):1682-6.

48. Martin SL, Kirkner GJ, Mayo K, Matthews CE, Durstine JL, Hebert JR. Urban, rural, and regional variations in physical activity. J Rural Health. 2005;21(3):239-44.

49. Baernholdt M, Yan G, Hinton I, Rose K, Mattos M. Quality of life in rural and urban adults 65 years and older: findings from the national health and nutrition examination survey. J Rural Health. 2012;28(4):339-47.

50. Ministry of Health, Labour and Welfare of Japan. Establishing "the Community-based Integrated Care System". 2015. https://www.mhlw. go.jp/english/policy/care-welfare/care-welfare-elderly/dl/establish_e.pdf

51. Christiansen LB, Cerin E, Badland H, Kerr J, Davey R, Troelsen J, et al. International comparisons of the associations between objective measures of the built environment and transport-related walking and cycling: IPEN Adult Study. J Transp Health. 2016;3(4):467-78.

\section{Publisher's Note}

Springer Nature remains neutral with regard to jurisdictional claims in published maps and institutional affiliations. 